Original paper UDC 165.62(045)

doi: $\frac{10.21464 / \mathrm{sp} 34210}{\text { Received: } 24 \text { July } 2019}$

\title{
Niels Weidtmann
}

Eberhard Karls Universität Tübingen, Forum Scientiarum, Doblerstraße 33, DE-72074 Tübingen niels.weidtmann@fsci.uni-tuebingen.de

\section{Welt als Phänomen}

\section{Anmerkungen zu Husserl, Heidegger und Rombach ${ }^{1}$}

\begin{abstract}
Zusammenfassung
Einen Beitrag über Welt als Phänomen zu schreiben, impliziert die Annahme, dass die Welt überhaupt ein Phänomen im Sinne der philosophischen Phänomenologie ist. Das ist aber gerade in der Phänomenologie selbst nicht unstrittig. Dem husserlschen Verständnis nach kann die Welt nämlich tatsächlich nicht selbst Phänomen sein, weil jedes Phänomen in einem Verweisungszusammenhang, d.h. in einem Horizont erscheint, die Welt selbst aber gerade nicht. Die Welt ist der äußerste Horizont und kann daher nicht über sich hinaus auf einen weiteren Horizont verweisen. Daher ist es keineswegs selbstverständlich zu behaupten, dass die Welt als Phänomen betrachtet werden kann. In meinem Beitrag werde ich nun allerdings noch über die Annahme, dass die Welt ein Phänomen ist, hinausgehen und zu zeigen versuchen, dass sie in Wirklichkeit das Phänomen par excellence ist. In gewisser Weise ist die Welt das einzige Phänomen überhaupt, weil sie nicht nur allem, was in ihr erscheint, seinen besonderen Sinn verleiht, sondern sich dabei zugleich selbst je anders, nämlich diesem besonderen Sinn entsprechend zeigt. Das will ich insbesondere mit Heidegger und Rombach zu zeigen versuchen. Auf diese Weise hoffe ich, zumindest einige Aspekte einer phänomenologischen Analyse von ,Welt“ offenzulegen.
\end{abstract}

\section{Schlüsselwörter}

Welt, Sinn, Phänomenologie, Edmund Husserl, Martin Heidegger, Heinrich Rombach, Maurice Merleau-Ponty

„Der Begriff der Welt bzw. das damit bezeichnete Phänomen ist das, was bisher in der Philosophie überhaupt noch nicht erkannt ist“", so Martin Heidegger. ${ }^{2}$ Deshalb sei ,die Aufklärung des Welt-Begriffs (...) eine der zentralsten Aufgaben der Philosophie“. Freilich, so fährt er fort und so fragen auch wir, wie kann das sein? Wie kann es sein, dass ausgerechnet das Phänomen Welt in der Philosophie bislang ,überhaupt noch nicht" richtig gesehen und behandelt worden ist? Es ließe sich doch einwenden, dass die Philosophie und mit ihr die Wissenschaften schon immer nach den weltlichen Dingen, nach der Natur fragen. Freilich, Welt meint nicht das Innerweltliche. Aber: meint sie

Die Einleitung und die Teile 1-3 dieses Textes entsprechen einer gekürzten und in Teilen überarbeiteten Fassung von: Niels Weidtmann, „Heideggers ontologische Wende der Phänomenologie. Anmerkungen zum Verhältnis von Sinn und Sein“, in: Harald Seubert (Hg.), Neunzig Jahre Sein und Zeit. Die fundamentalontologische Frage nach dem Sinn von Sein, Alber, Freiburg - München 2019, S. 120-139. Teil 4 ist bislang unveröffentlicht.

2

Martin Heidegger, Grundprobleme der Phänomenologie, GA Bd. 24, Klostermann, Frankfurt/M 1975, S. 234. 
etwas anderes? Nein, denn ein solches ,etwas ' wäre selbst ein innerweltliches anderes. Die Welt geht nicht im Innerweltlichen auf, sie ist auch nicht die Summe alles Seienden. Und doch, wo anders sollte sie sich denn zeigen als am innerweltlich Seienden? Wie kann es dann aber sein, dass Welt bislang nicht erkannt ist? Hat sie sich möglicherweise noch gar nicht als das gezeigt, was sie ist? Wie aber wäre das möglich, liegt Welt doch allem anderen, das sich zeigt, immer schon voraus?

In den folgenden Überlegungen möchte ich die These stark machen, dass Welt deswegen noch nicht eigentlich gesehen ist, weil sie selber erst zur Welt kommen muss. Sie ist nicht einfach da, ebenso wenig ist sie freilich bloß entzogen. Vielmehr muss sie zur Erscheinung kommen und zur Erscheinung gebracht werden - und das in einem ontologischen, ja genetischen Sinn. Darin erst ist sie Phänomen.

Phänomenologie meint das Aufmerken auf das Zur-Erscheinung-kommen von Wirklichkeit oder, wie Heidegger es in Sein und Zeit sagt:

„Das, was sich zeigt, so wie es sich von ihm selbst her zeigt, von ihm selbst her sehen lassen.“3

Es würde der Phänomenologie freilich nicht bedürfen, wären die Phänomene überall und immer klar ersichtlich. Das aber sind sie nicht. Stattdessen ist es sogar so, dass die Phänomene „zunächst und zumeist nicht gegeben sind“, wie Heidegger sagt. ${ }^{4}$ Sie sind deshalb ,zunächst und zumeist nicht gegeben“", weil sie sich ,zunächst und zumeist" nicht von sich selbst her, sondern von anderswoher zeigen. Etwa, wie Pascal sagt, von der Gewohnheit her. Dann sehen und verstehen wir die Dinge so, wie sie üblicherweise gesehen und verstanden werden, ohne sie eigens zu befragen und uns selbst von ihnen ansprechen zu lassen; wir leben unser Leben auf die Weise, wie Generationen vor uns ihr Leben gelebt haben, ohne es selber zu verantworten. Oder, um ein weiteres Beispiel Pascals aufzugreifen, das Heideggers Wort, Wissenschaft denke nicht, ${ }^{5}$ ebenso vorgreift, wie sich seine Analyse der Gewohnheit in der Verfallenheit an das Man widerspiegelt: Wir wenden unseren Kopf ein wenig und erlernen die wenigen und leicht zu begreifenden Prinzipien wissenschaftlichen Denkens, um die Wirklichkeit fortan diesen Prinzipien folgend erklären zu können. ${ }^{6}$ So oder so lassen wir die Wirklichkeit nicht selber sprechen und sehen sie nicht so, wie sie sich von sich selbst her zeigt. Verschiedene Phänomene können nie gleich-gültig sein und folglich in einem nivellierten Wirklichkeitsverständnis auch nicht zur Erscheinung kommen, weil sie ihre je-eigene Sinndimension mit aufscheinen lassen.

Die Phänomenologie macht es sich deshalb zur Aufgabe, das Seiende nicht geradehin als das zu nehmen, als das wir es in der durch den Blick der Gewohnheit oder der Wissenschaftlichkeit immer schon vorstrukturierten Welt vorfinden, sondern daraufhin zu befragen und sehen zu lernen, was in ihm von ihm selbst her, und das heißt dann eben von seiner eigenen Wirklichkeitsstruktur bzw. seiner eigenen Weltlichkeit her zur Erscheinung kommt.

Merleau-Ponty beschreibt das, worauf es mir hier wesentlich ankommt, sehr schön am Beispiel des für die Phänomenologie so naheliegenden Phänomens des künstlerischen Sehens. ${ }^{7}$ Er spricht davon, dass sich das Sehen ,aus der Mitte der Dinge heraus" ereignet, anstatt den Dingen bloß gegenüberzutreten. ${ }^{8}$ Das ,Rätsel der Sichtbarkeit“, ${ }^{9}$ von dem er spricht, lässt sich nur fassen, wenn der Mensch am Geschehen der Sichtbarwerdung teilhat, wenn Sehen und Sichtbarkeit in ihrer wechselseitigen Konstitution erfahren werden. Die Künstler sind diejenigen, die so zu sehen vermögen, dass darin die Zusammengehörigkeit von Sehen und Sichtbarkeit mitgesehen wird; die das Sehen 
als das Geschehen der Sichtbarwerdung erfahren, an dem sie teilhaben. Kunst, das sagt schon Klee, ,gibt nicht das Sichtbare wieder, sondern macht sichtbar". ${ }^{10}$ Sie macht dies, indem sie das Sichtbare auf seine Sinndimension hin befragt und es aus ihr heraus von neuem sichtbar werden lässt. Das Sichtbare ist nicht einfach dies oder das, sondern es ist die Selbstaussage einer ganzen Sinndimension, die sich niemals auf eine einzelne Aussage reduzieren lässt. Tatsächlich spricht Merleau-Ponty ausdrücklich von einer solchen Dimension, die im einzelnen Ding sichtbar wird:

„Jedes sichtbare Ding wirkt trotz seiner Individualität auch als Dimension, weil es sich als Ergebnis einer Aufspaltung (déhiscence) des Seins darbietet. Das bedeutet letzten Endes, daß es dem Sichtbaren eigentümlich ist, im strengsten Sinne des Wortes durch ein Unsichtbares gedoppelt zu sein, das es als ein gewissermaßen Abwesendes gegenwärtig macht." "11

Heidegger macht auf etwas ganz Ähnliches aufmerksam, wenn er in den KleeNotizen davon spricht, dass Klee der Maler des Zwischen ist. Das Zwischen meint jenen Ort, der zwischen dem Sichtbaren und dem, was im Sichtbaren sichtbar wird, zwischen Sein und Seiendem liegt. Dieses Zwischen ist der Ort des Wirklichkeitsgeschehens, in ihm gehören das Sichtbare und sein Sinn, Sein und Seiendes unmittelbar zusammen, und deswegen lässt sich das Sichtbare nur von ihm her in seiner eigentlichen Wirklichkeit sehen. Ein solches Sehen ordnet die Phänomene nicht in ein von außen an sie herangetragenes, wissenschaftliches oder alltägliches Weltverständnis ein, sondern erschließt sie von ihrer eigenen Strukturierung der Welt her. So bedeutet Phänomenologie letztlich immer zugleich mit dem Eintauchen in die Phänomene das Eintauchen in die Welt der Phänomene. Von sich her zeigen als das, was es ist, so lautet deshalb die These meines Beitrags, kann sich etwas nur in seiner Welt. Das Sich-zeigen des Phänomens ist darum zugleich der Aufgang seiner Welt. Welt ist Phänomen im Wortsinne. Dem möchte ich im Folgenden in vier Schritten nachgehen.

\section{Welt als Horizont}

Husserl zeigt in den Ideen II, was es heißt, dass ein Gegenstand nur auf dem Boden einer ganzen Sinndimension als der Gegenstand erfasst werden kann, der er ist:

3

Martin Heidegger, Sein und Zeit, GA Bd. 2, Klostermann, Frankfurt/M. 1977, S. 46.

Ebd., S. 48.

5

Martin Heidegger, Was heißt denken?, GA Bd. 8, Klostermann, Frankfurt/M. 2002, S. 9.

Blaise Pascal, Gedanken, Hg. von Jean-Robert Armogathe, übersetzt von Ulrich Kunzmann, Philipp Reclam, Stuttgart, jun. 1997, Nr. 512 (nach Lafuma).

Maurice Merleau-Ponty, „Das Auge und der Geist", Das Auge und der Geist. Philosophische Essays, Hg. von Christian Bermes, Meiner, Hamburg 2003, S. 275-317, S. 280.
8

Vgl. dazu Niels Weidtmann, „Das Sichtbarwerden des Unsichtbaren. Anmerkungen zum Verhältnis von Bild und Wirklichkeit", in: Georg Stenger, Sergej Seitz (Hg.), Kraft, Macht und Gewalt der Bilder, Springer, Cham 2018, S. 71-89.

9

M. Merleau-Ponty, ,Das Auge und der Geist“", S. 284.

10

Paul Klee, „Schöpferische Konfession“, in: Kasimir Edschmid (Hg.), Tribüne der Kunst und Zeit. Eine Schriftensammlung, Erich Reiß Verlag, Berlin 1920, S. 28-40, hier 28.

11

M. Merleau-Ponty, ,Das Auge und der Geist“", S. 313. 
„Im Wahrnehmen ist nun dieser Tisch gegeben, aber in jeweils bestimmter Weise gegeben. Das Wahrnehmen hat seinen Wahrnehmungssinn, sein Vermeintes, wie es gerade Vermeintes ist, und in diesem Sinn liegen Anweisungen, liegen unerfüllte Vordeutungen und Zurückdeutungen, denen wir nur zu folgen haben. (...) Nur wenn man das Dingnoema, sozusagen die Dingmeinung, indem man sie nach allen Richtungen zu entfaltender Gegebenheit bringt, selbst befragt und von ihr sich im Vollzug ihrer Anweisungen Antwort geben läßt, gewinnt man die Wesenskomponenten der Dinglichkeit wirklich und die notwendigen Wesensverflechtungen, ohne die Dingvermeintes überhaupt nicht gedacht werden kann." ${ }^{12}$

Worauf Husserl hier aufmerksam macht, ist, dass wir im einzelnen Wahrnehmungsakt immer sehr viel mehr wahrnehmen, als reell in ihm gegeben ist. Wir nehmen einen Tisch wahr, obwohl uns dieser in der Wahrnehmung immer nur eingeschränkt oder, wie Husserl sagt, abgeschattet gegeben ist. Wir können den Tisch nur deshalb wahrnehmen, weil wir die Abschattung immer schon als Abschattung eines Sinnganzen verstehen. Da wir den Tisch als solchen nie in der Weise wahrnehmen können, dass er als ganzer reeller Bestandteil der Wahrnehmung wäre, setzt die Wahrnehmung also grundsätzlich voraus, dass wir sie vor dem Hintergrund eines Erfahrungszusammenhangs verstehen. Erst von diesem Erfahrungszusammenhang her wird das in der Wahrnehmung Gegebene als Sinnganzes vermeint. Der Wahrnehmungsgegenstand ist deswegen notwendig vermeinter oder intentionaler Gegenstand. Noch einmal am Beispiel der Tischwahrnehmung: Der Tisch wird nicht deshalb als Tisch wahrgenommen, weil er adäquat repräsentiert wird, sondern weil er vor dem Hintergrund eines in der Wahrnehmungserfahrung konstituierten Sinnzusammenhangs gesehen wird. Für diesen Erfahrungs- bzw. Sinnzusammenhang verwendet Husserl den Begriff des Horizonts. Die Aufgabe der Phänomenologie liegt demnach in der Aufklärung von Horizonten. Sie ist deskriptiv, aber sie beschreibt nicht das an der Oberfläche der Wirklichkeit vorfindbare Seiende, sondern den Verweisungszusammenhang, in dem dieses Seiende steht und der es erst zu dem macht, was es ist. Phänomenologie ist Horizontanalyse und als solche Konstitutionsforschung.

Die Welt, in der wir leben, die „Lebenswelt“, ist Husserl zufolge also im Wortsinne Wahrnehmungs- bzw. allgemeiner Erfahrungswelt und keine bloße Ansammlung von Seiendem. Die Erfahrung ist dabei immer aus früheren Erfahrungen motiviert und bildet notwendiger Weise einen kontinuierlichen Erfahrungszusammenhang. Zwar steht sie grundsätzlich der Bestätigung offen, dabei kann sie aber nur bestätigt oder widerlegt, nicht aber beliebig angepasst werden. Eine korrigierte Erfahrung kann stattdessen ihrerseits nur dadurch zustande kommen, dass das im Erfahrungsakt Gegebene im Horizont anderer zuvor gemachter Erfahrungen gesehen wird. Husserl kann die Lebenswelt in der Krisis-Schrift deshalb als geschichtlich sich entwickelnde Erfahrungswelt beschreiben. Erfahrungen werden intersubjektiv geteilt, sedimentieren und bilden so den Boden für weitere Erfahrungen. ${ }^{13}$ Die Welt wird auf diese Weise immer erfahrungsgesättigter und das heißt eben auch, sie wird immer evidenter, ihr Sinn immer offensichtlicher und die Menschheit darum zugleich immer einiger. Es ist ein unglaublicher Fortschrittsoptimismus, der da hinter Husserls Analyse einer tiefgreifenden Krise des europäischen Geistes hervorkommt.

Während die transzendentale Erfahrung selbst nicht weiter hinterfragbar ist - in ihr findet Husserl jene ,apodiktische Evidenz“ ${ }_{14}^{14}$ die die Phänomenologie anzielen muss, will sie erste Philosophie sein - kann dagegen alles in der Erfahrung Konstituierte, und damit die Welt im Ganzen, nicht in der gleichen Weise apodiktisch evident sein, sondern muss sich in unendlich fortlaufender Erfahrung bewähren. Das ist der Ansatzpunkt Heideggers. Er macht gegen 
Husserls Analyse geltend, dass sich die Wahrnehmung eines Gegenstandes wie beispielsweise die des Tisches durch die Analyse des zugehörigen Horizonts zwar entscheidend erhellen lässt, dass sie aber voraussetzt, dass dieser Horizont seinerseits geklärt, und das heißt vor dem Hintergrund eines weiter ausgreifenden Horizonts verstanden ist. So vermag der Horizont ,Tisch ' der Einzelwahrnehmung nur dann Sinn zu verleihen, wenn dem zuvor klar ist, worin der Sinn eines Tisches liegt. Das setzt etwa einen Horizont von Wohnen oder möglicherweise auch einen religiösen Horizont voraus, da sich der Sinn von Tisch vermutlich ursprünglich dem Opferaltar verdankt. Diese Horizonte setzen ihrerseits weitere Horizonte voraus, die geklärt sein müssen, bevor das in der Einzelwahrnehmung Wahrgenommene als ein Tisch verstanden werden kann. Horizonte verweisen auf andere Horizonte nicht nur im Sinne weitergehender Erfahrung, sondern sie setzen einander auch voraus, um überhaupt sinnstiftend wirken zu können. So liegt Heideggers Einwand gegen Husserl, dass Welt als der äußerste, alle anderen umgreifende Horizont immer schon verstanden sein muss, damit sich überhaupt verschiedene Verständnishorizonte auffächern können, in der Konsequenz der husserlschen Phänomenologie. Zugleich wird deutlich, dass Welt gar nicht als Horizont verstanden werden darf, würde das doch einen weiteren, den Welthorizont klärenden Horizont voraussetzen. Wenn Welt kein Horizont ist, dann kann sie auch nicht transzendental konstituiert sein. Und doch kann das Ego Erfahrungen nur machen, weil es sich immer schon in einem Weltverständnis aufhält. Damit stößt Heidegger auf die strukturelle Zusammengehörigkeit von Ego und Welt. Er folgt Husserl darin, dass Welt nicht die Summe des geradehin Vorfindbaren meint, sondern grundsätzlich verstandene, aufgefasste, vermeinte Welt ist. Sie ist eben nur nicht transzendental konstituiert, weil jede solche Konstitution das Verstehen von Welt schon voraussetzt. Heidegger zeigt, dass dem Ego Welt immer schon zugehört und fasst diese Zugehörigkeit in der Struktur des „In-der-Welt-seins“. Sehr deutlich wird die Sollbruchstelle zwischen Husserl und Heidegger an Heideggers Kommentierung des husserlschen Encyclopaedia Britannica-Artikels zum Stichwort Phänomenologie. Heidegger notiert an entsprechender Stelle:

„Gehört nicht eine Welt überhaupt zum Wesen des reinen Ego?“15

Wenn Phänomenologie meint, die Sinndimension im Seienden zur Erscheinung kommen zu lassen, dann bedeutet das nun also: Das einzelne Seiende ist nur dann richtig gesehen, wenn in ihm Welt mitgesehen wird. Das ist mit der ontologischen Wende der Phänomenologie gemeint; die Phänomenologie darf sich nicht wie noch in der Reduktion Husserls auf die transzendentale Erfahrung vom Sein der Welt abwenden, sondern sie muss sich umgekehrt

12

Edmund Husserl, Ideen zu einer reinen Phänomenologie und phänomenologischen Philosophie. Zweites Buch: Phänomenologische Untersuchungen zur Konstitution, Hua IV, hg. von Marly Biemel, Martinus Nijhoff, Den Haag 1952, S. 35.

13

Ich gehe hier nicht näher auf die Intersubjektivitätsproblematik ein. Vgl. dazu v.a. die 5. Meditation in Edmund Husserl, Cartesianische Meditationen und Pariser Vorträge, Hua I, hg. von Stephan Strasser, Martinus Nijhoff, Den Haag ${ }^{2}$ 1963; außerdem Edmund
Husserl, Zur Phänomenologie der Intersubjektivität, Hua XIII, XIV und XV, hg. von Iso Kern, Martinus Nijhoff, Den Haag 1973.

14

E. Husserl, Cartesianische Meditationen und Pariser Vorträge, S. 56.

15

Edmund Husserl, Phänomenologische Psychologie. Ergänzungen, Hua IX, hg. von Walter Biemel, Martinus Nijhoff, Den Haag 1968, S. 273 f. 
gerade zur Welt hinwenden. Das Mitsehen von Welt im einzelnen Seienden bedeutet, das Seiende eigens in seinem Sein sehen zu lernen und solches Sein nicht einfach vorauszusetzen.

\section{2. „In-der-Welt-sein“}

Husserl hatte die Frage nach dem Sein der Welt bewusst ausgeklammert, um sich ganz darauf besinnen zu können, was uns in der Erfahrung gegeben ist. Dadurch musste einerseits der Sinn der Erfahrungen als vom Sein der Welt unabhängig erscheinen, andererseits konnte die transzendentale Erfahrung als letzter Konstitutionsgrund verstanden werden, der sich nicht weiter erforschen ließ. Für Husserl konnte das evidente Verstehen von Welt darum auch bloß eine Idee darstellen; wenn alle nur möglichen Erfahrungen gemacht sind, und das sind unendlich viele, dann erst steht die Welt im Ganzen klar und deutlich vor uns. Für Heidegger dagegen ist das Weltverstehen jene Offenheit, die Erfahrungen überhaupt erst möglich macht. Mit der Rückführung der transzendentalen Erfahrung, die das Weltverständnis konstituiert, auf die Struktur des „In-der-Welt-seins“" stellt sich deshalb auch die Frage nach dem Sein von neuem. Während das husserlsche Ego das Sein in der Erfahrung bloß vermeint, vollzieht das als „In-der-Welt-sein“ verstandene Dasein in der Erfahrung sein eigenes Sein. Damit wird die Frage nach dem Sein zur drängendsten. Sie muss zunächst geklärt sein, soll überhaupt irgendetwas zum Sinn von Erfahrungen gesagt werden können. Die ursprüngliche Sinnfrage ist deshalb die Frage nach dem Sein.

Wenn der Sinn einer Sache erfasst werden soll, dann reicht es eben nicht aus, den Horizont aufzuklären, vor dem sie zur Erscheinung kommt, sondern dann muss das ihr eigene Sein aufgewiesen werden. Dieses ist es denn auch, was „zunächst und zumeist" verborgen bleibt und deswegen phänomenologisch gehoben werden muss. Damit bricht die Phänomenologie erst eigentlich in die Wirklichkeit ein. Etwas ist phänomenologisch erst dann richtig erfasst, wenn es nicht nur als sinnvoll erscheint, sondern sinnvoll ist. Das ist evident. Sinnvoll sein kann etwas aber nur dann, wenn es von der Bewandtnisganzheit der Welt her bestimmt ist; und dementsprechend kann es als sinnvoll erfasst werden nur dann, wenn zuvor Welt verstanden ist. Die Frage nach dem Sein lässt sich folglich nur vom Weltverstehen her beantworten. Ja, Weltverstehen ist geradezu die Weise, wie Sein anwest. Darum kann Heidegger sagen, dass zum Da-Sein offenbar Weltverstehen gehört. Das Dasein ist in die Struktur des „In-der-Welt-seins“ auseinander gelegt. Nicht-daseinsmäßig Seiendes kann dagegen nur in der Welt vorkommen. Die Seinsweise des Daseins gründet deshalb alle weiteren Seinsweisen, darum spricht Heidegger von Fundamentalontologie.

Das Dasein wird in Sein und Zeit als dasjenige Seiende beschrieben, dem es in seinem Sein um dieses Sein selber geht. Das Dasein vollzieht sein eigenes Sein und ist immer schon über sich hinaus zu sich selbst unterwegs; und das ganz konkret dadurch, dass es sein eigenes Weltverstehen auslegt. Rombach kann deshalb mit Blick auf Heideggers Daseinsanalyse sagen, dass sich das Dasein als Welt entgegenkommt. ${ }^{16}$ Freilich erkennt es sich darin zumeist eben nicht und verliert sich deshalb in der und an die Welt. ${ }^{17}$ Ich will hier nicht weiter auf die Unterscheidung von Uneigentlichkeit und Eigentlichkeit, den Ruf des Gewissens, das Vorlaufen zum Tode und das Phänomen der Entschlossenheit eingehen. Im Zuge unseres Gedankengangs ist die entscheidende Frage, was genau Welt bedeutet. Das Dasein spannt im auslegenden Vollzug seines 
Weltverstehens jene Welt auf, in der das nicht-daseinsmäßig Seiende begegnen kann. Es öffnet also einen Raum, ja es ist wesentlich Offenheit, Auseinander, Aufriss. Heidegger zeigt das in seiner Analyse der Zeitlichkeit des Daseins. Das Dasein ist im Entwurf zukünftig und im Geworfensein gewesen, und das in der Gegen-wärtigkeit seines in den Entwurf Geworfenseins. So in die Dimensionen der Zeit auseinandergelegt ist das Dasein wesentlich Offenheit und als Offenheit vermag es die anderen Seinsweisen zu gründen. Der Sinn des Seins des Daseins ist darum die Zeit. Die Zeit ist das Auseinander des Seins, das Da des Seins, seine Lichtung, die allein das Zur-Erscheinungkommen des Seienden ermöglicht. Das nicht-daseinsmäßig Seiende kommt denn auch nur in der vom Dasein eröffneten Welt zur Erscheinung.

Damit ist auch nochmals gesagt, worin der entscheidende Gewinn der ontologischen Phänomenologie Heideggers gegenüber der transzendentalen, auf Horizonte zielenden Phänomenologie Husserls liegt. Bei Husserl bleibt das Sein der Welt als bloßes ,Dass“ vorausgesetzt; nur deswegen kann es in der Reduktion auch eingeklammert werden. Heidegger dagegen zeigt, dass die Phänomenologie das Sein der Welt selbst befragen und in seinem Zur-Erscheinung-kommen aufweisen muss. Nur so kann die Phänomenologie ihre Aufgabe, ,das, was sich zeigt, so wie es sich von ihm selbst her zeigt, von ihm selbst her sehen [zu] lassen“, auch tatsächlich erfüllen. Die Daseinsanalyse hat zudem enthüllt, dass das Dasein, das wir je selber sind, selbst der Vollzug des Zur-Erscheinung-kommens des Seins ist. Der Mensch lebt also in jenem Zwischen, ja als jenes Zwischen, das Merleau-Ponty als das Reich der Malerei bezeichnet hat und das wir eingangs auch für die Phänomenologie in Anspruch genommen haben. Der Mensch, so ließe sich zugespitzt formulieren, ist Künstler und er betreibt auch nicht nur gelegentlich Phänomenologie, vielmehr ist er in allem, was er tut, eigentlich Phänomenologe, weil er durch sein eigenes Dasein dem Seienden zum Zur-Erscheinung-kommen verhilft.

Rombach weist allerdings darauf hin, dass sich in Sein und Zeit auch einige Stellen finden, an denen Heidegger doch wieder so etwas wie das bloße „Dass“ des Seienden vorauszusetzen und damit in ein transzendentales Phänomenologieverständnis zurückzufallen scheint. ${ }^{18}$ Im Paragraphen 43 heißt es unter der Überschrift „Realität und Sorge“:

„Daß Realität ontologisch im Sein des Daseins gründet, kann nicht bedeuten, dass Reales nur sein könnte als das, was es an ihm selbst ist, wenn und solange Dasein existiert."19

Mit der Unterscheidung von Realität, die am Dasein hängt, und Realem, das unabhängig vom Dasein gegeben ist, möchte Heidegger, so Rombachs Vermutung, dem möglichen Vorwurf des Subjektivismus zuvorkommen. Und doch scheint er damit nur zu offenbaren, dass er selbst das Dasein als ein dem Realen gegenüberstehendes Subjekt missversteht. Die am Dasein hängende Realität, in der das Reale erscheint, ist dann aber nichts anderes als ein Horizont. In der Marburger Vorlesung vom Sommersemester 1927 lesen wir ganz ähnlich:

„Welt ist nur, wenn und solange ein Dasein existiert. Natur kann auch sein, wenn kein Dasein existiert." 20

16

Heinrich Rombach, Phänomenologie des gegenwärtigen Bewusstseins, Alber, Freiburg 1980, S. 139.

17

Ebd.
18

Ebd., S. 110.

19

M. Heidegger, Sein und Zeit, S. 280.

20

M. Heidegger, Die Grundprobleme der Phänomenologie, S. 241. 
Welt ist dann aber das, was von anderswoher an das Seiende herangetragen wird. In seinem Aufsatz „Vom Wesen des Grundes“ (1929) spricht Heidegger explizit von einem „Weltüberwurf“"

„Die Welt wird als die jeweilige Ganzheit des Umwillen eines Daseins durch dieses selbst vor es selbst gebracht. Dieses Vor-sich-selbst-bringen von Welt ist der ursprüngliche Entwurf der Möglichkeiten des Daseins, sofern es inmitten von Seiendem zu diesem sich soll verhalten können. Der Entwurf von Welt aber ist, imgleichen wie er das Entworfene nicht eigens erfasst, so auch immer Überwurf der entworfenen Welt über das Seiende. Der vorgängige Überwurf ermöglicht erst, dass Seiendes als solches sich offenbart. Dieses Geschehen des entwerfenden Überwurfs, worin sich das Sein des Daseins zeitigt, ist das In-der-Welt-sein. “21

Wo liegt da noch die Differenz zu Husserl? So verstanden ist die Welt nichts anderes als ein Horizont, in dem das Seiende zur Erscheinung kommt und einen geschichtlichen Sinn erhält. Das kann nicht Heideggers Intention sein. Umso mehr freilich zeigen sich offene Fragen. Allen voran die Frage nach dem Verhältnis von Welt und Sein, scheint Heidegger in den genannten Passagen doch zu sagen, der Sinn des Seins von nicht-daseinsmäßig Seiendem hinge davon $a b$, dass es in der vom Dasein entworfenen Welt erscheint, das bloße Sein des Seienden aber nicht. Was ist Sein, solange sein Sinn nicht entdeckt, solange es nicht von der Welt her verstanden ist?

\section{Welt und Sein}

Vermutlich muss Heidegger in Sein und Zeit an den Punkt kommen, das nicht-daseinsmäßig Seiende so, wie es an sich selbst ist, als unabhängig vom Weltverstehen des Daseins anzunehmen und gleichsam schon vorauszusetzen. Schließlich ist das Dasein selbst ein Seiendes, das im Verstehen von Welt seine eigene Seinsweise aufklärt. Darin liegt der hermeneutische Zirkel, und in diesem Sinne ist Heideggers ontologische Phänomenologie primär Hermeneutik. Wenn sich das Dasein auf dem Weg zu sich selbst als Welt entgegenkommt und darin das Sein des Seienden entdeckt, so ist das eben nicht gleichbedeutend mit dem Erscheinen des Seins selbst qua Seiendes, sondern offenbart lediglich das Sein desjenigen Seienden, das als sein eigenes Seinkönnen existiert. Es sagt deshalb nur etwas über das Sein des Daseins, nicht aber über das Sein des Seienden aus. Der Ansatz der Fundamentalontologie erweist sich darin, dass er ein einzelnes Seiendes zum Ausgangspunkt nimmt für das Erscheinen des Seins alles Seienden, als fraglich.

Heidegger hat das zweifellos selber gesehen und er hat gesehen, dass damit das Seinsdenken im Ganzen auf dem Spiel steht. Er hat nach Sein und Zeit darum nochmals neu angesetzt und die Welt nicht vom Dasein, sondern nun konsequent vom Sein her zu denken versucht. Indem er etwa in dem auf Sein und Zeit reflektierenden Vortrag Zeit und Sein aus dem Jahre 1962 vom Seinsgeschick spricht, denkt er das Sein und die Entdeckung des Seins qua Welt nicht mehr von der Zeitlichkeit des Daseins her, sondern fasst es in seiner eigenen Geschichtlichkeit. ${ }^{22}$ Es ist gerade die Geschichtlichkeit, die den ontologischen Charakter des Seins auszuweisen vermag: Da Sein nicht selber ein Seiendes ist, kann es eben auch nicht in einem Horizont, und sei dieser Horizont die Welt, zur Erscheinung kommen. Stattdessen kann es sich nur selbst entdecken - und zwar so, dass es in der Entdeckung fortwährend selbstentdeckend bleibt. Das Sein wird also nicht vom Dasein entdeckt, sondern es entdeckt sich selbst, ohne dass solche Entdeckung je abschließend sein könnte. Die Unabschließbarkeit der Selbstentdeckung des Seins begründet die Geschichte. Das Sein kann sich nur so entdecken, dass es sich als Ent- 
deckendes allein im Entdeckten zeigt, als Entdeckendes aber selbst zurückhält. In diesem Sinne spricht Heidegger davon, dass sich das Sein epochal „schickt".

„Seinsgeschichte heißt Geschick von Sein, in welchen Schickungen sowohl das Schicken als auch das Es, das schickt, an sich halten mit der Bekundung ihrer selbst. An sich halten heißt

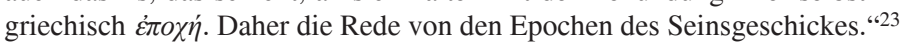

Heidegger denkt das Seinsgeschick in Zeit und Sein bekanntlich in enger Verschränkung zum „lichtend-verbergenden Reichen“ der Zeit, die im Zusammenspiel der Zeitdimensionen jenen Zeit-Raum öffnet, als welcher das geschickliche Sein anwest. Zeit und Sein gehören im „Ereignis“ zusammen und zwar so, dass sich in ihm das Sein als Anwesen ereignet. Im Ereignis stoßen wir also nicht auf eine das Sein begründende Dimension, sondern vernehmen das Sein in seiner Selbstaussage.

Es ist das Sein selbst, das sich entdeckt. Damit erst ist das Horizontdenken wirklich überwunden und wird die Phänomenologie wahrhaft ontologisch. Wenn Heidegger in dem erwähnten Aufsatz Zeit und Sein sagt, der Satz „Es gibt Sein“" sage so viel wie „Sein gibt Sein“, ${ }^{24}$ dann ist dies nichts anderes als die Grundstruktur aller Ontologie, die nun nicht mehr substanzialistisch wie noch in den überlieferten Begriffen von Ousia, Energeia und Substantia, sondern konsequent phänomenologisch, und das heißt hier vom eigenen Hervorgang her verstanden wird. Der Satz „Sein gibt Sein“ kann deshalb nur dann als Grundstruktur der Ontologie verstanden werden, wenn man ihn von innen heraus erfasst, was nichts anderes bedeutet, als dass er nur verstanden werden kann, wenn man selbst mitten im Seinsgeschehen steht. Und genau das ist der Fall. Der Mensch steht in der Lichtung des Seins und er ver-steht sie als Welt. Die Offenheit der Lichtung muss aus(einander)-gehalten werden, die Lichtung muss tatsächlich etwas erhellen, damit Sein anwesen kann; bzw. umgekehrt, Sein west dadurch an, dass der Mensch im Offenen der Lichtung steht und seine Welt aus der Helle der Lichtung heraus versteht. Heidegger spricht deshalb vom Menschen als dem „Hirt des Seins“. 25

\section{Welt und Welten}

Im Lichtungsgeschehen erscheint das Sein als Offenheit. Offenheit aber erscheint nur an anderem, sie ist grundsätzlich Offenheit für etwas, es gibt keine absolute Offenheit. Die Offenheit, als welche das Sein aufgeht, erscheint am Menschen und ist Offenheit für den Menschen, insofern der Mensch in die Lichtung hinaussteht. Der Mensch ist der „Hirt des Seins“, weil sich das Sein nur dann als Lichtung schicken kann, wenn der Mensch in diese Lichtung hinaussteht, d.h. wenn er sich selbst von der Offenheit der Lichtung her versteht und sie damit überhaupt erst zu jener Offenheit macht, in der sich das Seiende zu zeigen vermag. Weil der Mensch in die Lichtung hinaussteht, kann ihm

21

Martin Heidegger, ,Vom Wesen des Grundes“, Wegmarken, GA 9, Klostermann, Frankfurt/M. 1976, S. 158 (Hervorhebung im Original).

22

Martin Heidegger, „Zeit und Sein“, Zur Sache des Denkens, GA Bd. 14, Klostermann, Frankfurt/M. 2007, S. 3-30.
23

Ebd., S. 13.

24

Ebd., S. 23.

25

Martin Heidegger, „Brief über den ,Humanismus"“, Wegmarken, GA Bd. 9, Klostermann, Frankfurt/M. 1976, S. 342. 
das Seiende in der Lichtung begegnen. Was aber heißt das, dass das Seiende in der Lichtung begegnet? Seiendes, das in der Lichtung begegnet, erscheint „im Lichte des Seins (...) als das Seiende, das es ist", so Heidegger im Humanismusbrief. ${ }^{26}$ Von sich aus steht das Seiende nicht in der Wahrheit des Seins. Selbst Tiere sind „,zwar je in ihre Umgebung verspannt, aber niemals in die Lichtung des Seins, und nur sie ist ,Welt", frei gestellt" “. ${ }^{27}$ Dass das Seiende in der Lichtung begegnet, meint dann offenbar, dass es aus seinem Verspanntsein in die Umgebung gelöst und ins Offene der Welt gestellt wird.

An dieser Stelle müssen wir innehalten und dem Gesagten nach-denken. Was ist gesagt? Zum einen meint Lichtung des Seins offenbar Welt. Welt aber immer als geschichtliche, das Sein, das sich in ihr lichtet, eigens zurückhaltende, d.h. epochale Welt. Diese epochale Welt ist zudem Welt für den Menschen, sie entdeckt die je geschichtliche Seinsmöglichkeit des Menschen. Schließlich ist drittens gesagt, dass der Mensch das Seiende aus seinem Verspanntsein in die Umgebung löst. Dadurch, dass er ihm im Offenen der Welt begegnet, ermöglicht er erst, dass das Seiende als das, was es ist, erscheint. All das ließe sich von Sein und Zeit her so verstehen, dass das Seiende seinen Sinn von der „Bewandtnisganzheit“ der Welt her erhält, die ihrerseits die vom Menschen ergriffene, ihm eigene Seinsmöglichkeit darstellt. Der Unterschied zur Daseinsanalyse aus Sein und Zeit liegt allein darin, dass die dem Menschen eigene Seinsmöglichkeit nicht im entschlossenen Ergreifen des eigenen Entwurfs liegt, sondern vom Sein als dessen eigene Lichtung geschickt ist. Abgesehen davon, dass bislang völlig ungeklärt ist, worin die konkrete geschichtliche Gestalt der Welt begründet ist - schließlich kann das Seinsgeschick nicht zufällig sein, - bleiben bei einer an die Daseinsanalyse angelehnten Interpretation auch die bereits gegen die Daseinsanalyse ins Feld geführten Einwände bestehen. Wenn das Seiende dadurch zu dem wird, was es ist, dass es von einer vom Sein entdeckten und vom Menschen entgegengenommenen „Bewandtnisganzheit“ der Welt her verstanden wird, dann meint Welt nichts anderes als Horizont.

Ich glaube aber, dass wir Heidegger im Humanismusbrief und natürlich auch in Zeit und Sein anders verstehen dürfen. Anders als noch in Sein und Zeit beschrieben, lässt sich die als Lichtungsgeschehen des Seins verstandene Welt nicht mehr als eine Bewandtnisganzheit auslegen, von der her das Seiende seinen je eigenen Sinn erhält. Vielmehr ist die Lichtung, von der Heidegger sagt, dass nur sie Welt ist, bloße Offenheit. Sie gibt keinen spezifischen epochalen Seinssinn vor. Sie gewinnt diesen erst darin, dass der Mensch in die Lichtung hinaussteht und das Offene der Welt als sein Eigenstes, seine ur-eigene Seinsmöglichkeit ergreift. Wie kann die Welt ihren epochalen Sinn dadurch gewinnen, dass sich der Mensch in das Offene der Welt stellt? Dadurch dass sich der Mensch in das Offene der Welt stellt, vermag er auch dem Seienden in der Offenheit der Welt zu begegnen. Das heißt nun aber gerade nicht, dass er das Seiende vom Horizont der Welt her versteht, sondern dass er das Seiende selber in die Lichtung stellt, was nichts anderes bedeuten kann, als dass er das Seiende selber lichtet und es damit von Seinsmöglichkeiten her versteht, die nicht von außen an das Seiende herangetragen werden. Solche „Möglichkeiten“ sind allerdings missverstanden, wenn sie als ihrer Verwirklichung harrende Formen vorgestellt werden, die bereits im Seienden vorliegen. Ein solches Verständnis von Möglichkeit ist von der aristotelischen Unterscheidung zwischen Möglichkeit im Sinne von potentia und Wirklichkeit im Sinne von actus her gedacht. ${ }^{28}$ Die Entfaltung der potentia bringt aber lediglich jenes Potential zu voller Geltung, über das das Seiende in einem gegebenen Sinnzusammenhang bereits verfügt. Der Mensch versteht 
das Seiende dagegen auch von solchen Seinsmöglichkeiten her, die das Seiende selbst weit übersteigen, die aber dennoch nicht von anderswoher an das Seiende herangetragen werden, sondern aus der Begegnung des Menschen mit dem Seienden gewonnen sind. Mit Merleau-Ponty, aber ebenso mit Heidegger ließe sich von der „Dimension“ sprechen, die mit dem Seienden dann aufgeht, wenn es nicht von anderswoher, sondern in seinem eigenen Sinn zur Erscheinung und zur Entfaltung gebracht wird. ${ }^{29}$ Solche Dimension ,ermisst“ das Seiende als das, was es von sich selbst her ist. Das Auszeichnende des Menschen liegt demnach darin, dass er das Seiende nicht als das nimmt, als das er es in alltäglichen Zusammenhängen vorfindet, sondern es stattdessen auf Möglichkeiten hin anspricht, die sich erst durch den Aufgang der das Seiende ermessenden Dimension ergeben. Heidegger scheint mir auf eine solche Lesart bereits ganz zu Beginn des Humanismusbriefs hinzudeuten, wenn er sagt, dass Handeln eigentlich „Vollbringen“ meint im Sinne von etwas ,in die Fülle seines Wesens entfalten" ${ }^{30}$ Das gilt zwar im Besonderen für den Bezug des Menschen zum Sein und damit für das Denken, in dem sich das Sein ausspricht, es gilt aber von diesem Seinsbezug her gesehen eben auch für alles andere Handeln des Menschen. Der Mensch begegnet dem Seienden in der Offenheit der Welt so, dass er das Seiende „in die Fülle seines Wesens“ entfaltet.

Freilich bleibt auch in dieser Lesart des späten Heidegger eine entscheidende Frage offen. Heidegger scheint die Welt als Lichtung und damit als prinzipielle Offenheit vorauszusetzen, damit der Mensch dem Seienden so begegnen kann, dass er es ,in die Fülle seines Wesens“ entfaltet. Was aber soll eine solche, der Begegnung von Seiendem vorausgehende, prinzipielle Offenheit sein? Es gehört zum Wesen des Menschen, dem Seienden lichtend zu begegnen - darin ent-spricht er dem Sein. Aber was soll die Lichtung sein, wenn sie nicht Seiendes lichtet? Wenn Lichtung aber nur dort ist, wo Seiendes in der Begegnung mit dem Menschen gelichtet wird, dann hat Lichtung in jedem einzelnen Fall einen ganz konkreten, nie aber einen nur prinzipiellen Sinn. Offenheit qua Offenheit gibt es nicht. Es gibt nur die Offenheit für etwas. Man kann den Menschen deshalb tatsächlich von seinem Offensein für die Offenheit der Welt her verstehen. Das Offensein ist dann aber etwas spezifisch Menschliches, wohingegen die Offenheit, für die der Mensch offen ist, eben immer konkret ist und vom jeweiligen Seienden, das „,in die Fülle seines Wesens“ zu entfalten ist, her verstanden werden muss. Der Mensch hat Welt, aber welche Welt er hat, entscheidet sich daran, welchem Seienden - und vielleicht noch viel wichtiger, wie er diesem Seienden - begegnet. Das darf nun natürlich nicht als Rückfall in eine anthropologische oder ontische Auffassung verstanden werden. Welt liegt niemals in der Verfügung des Menschen. Es geht einzig darum zu sehen, dass die Begegnung des Menschen mit Seiendem immer öffnend ist, nie aber in zuvor schon gegebener Offenheit stattfindet. Offenheit kann nicht vorausgesetzt werden, ohne dadurch ihrer eigenen Offenheit verlustig zu gehen. Offenheit kann nur als Öffnung geschehen. Heidegger denkt das im Aletheia-Geschehen ebenso wie

26

Ebd., S. 330.

27

Ebd., S. 326.

28

Ebd., S. 316 f.
29

Vgl. FN 10 und Martin Heidegger, „Die Sprache“, in: Unterwegs zur Sprache, Neske, Stuttgart ${ }^{10} 1993$, S. 25.

30

M. Heidegger, „Brief über den ,Humanismus"“, S. 313. 
im Ereignis-Denken, aber er scheint nicht zu sehen, dass auch die Begegnung von Seiendem ein Öffnungsgeschehen sein muss und nicht in einer bereits ereignishaft stattgefundenen, irgendwie leer vorgestellten Offenheit statthaben kann. Das Sein lichtet sich in der Begegnung von Seiendem, nicht geht die Lichtung dieser Begegnung voraus.

In diesem Sinne spricht Rombach davon, dass Welt dort aufgeht, wo in der Begegnung von Mensch und Seiendem neue Seinsmöglichkeiten hervorgetrieben werden. ${ }^{31}$ Entscheidend ist zum einen zu sehen, dass sich etwas nur dann als das zeigen kann, was es ist, wenn es in diesem Sich-zeigen nicht bleibt, was es ist, sondern erst durch das Sich-zeigen zu dem wird, was es ist. Heidegger sieht das, zieht die neuen Seinsmöglichkeiten aber pauschal aus der epochalen Lichtung des Seins. Für Rombach ist es dagegen zum anderen entscheidend, dass sich die neuen Seinsmöglichkeiten aus der Begegnung des Menschen mit dem Seienden erst ergeben und immer sowohl den Menschen als auch das Seiende betreffen. Er spricht deshalb von „Konkreativität“. Das Seiende wird nicht dadurch gelichtet, dass es von einer Bewandtnisganzheit her verstanden wird, sondern immer nur dadurch, dass die in ihm liegenden Möglichkeiten entdeckt werden. Dieses, dass der Mensch die im Seienden liegenden neuen Seinsmöglichkeiten zu entdecken vermag, ist genau das, was Heidegger als das Hinausstehen des Menschen in die Lichtung des Seins bezeichnet - freilich nicht in die Lichtung eines wahlweise bloß abstrakten und blutleeren oder aber alles be- und überherrschenden Seins, sondern in die Lichtung der entdeckenden Begegnung des Menschen mit einzelnem Seienden. Es gibt tatsächlich so etwas wie die Welt der Handwerker, die Heidegger in Sein und Zeit als ein Beispiel heranzieht. Sie verdankt sich aber nicht dem Entwurf des Daseins, sondern ganz konkret den im Material, den Werkzeugen, der Werkstatt und dem Hand-Werk liegenden Möglichkeiten. Der Handwerker darf sein Material gerade nicht von einer vor-entworfenen Bewandtnisganzheit her verstehen, sondern muss im Gegenteil darauf achten, was das Material erfordert und ermöglicht, er muss auf das Material eingehen, um mehr aus ihm herausholen zu können, als offensichtlich in ihm steckt. Der Handwerker muss im Holz, im Metall, im Kunststoff wohnen, nicht darf er das Holz, das Metall, den Kunststoff in eine ihnen äußerliche Lichtung stellen. Das beste Beispiel für einen solchen Umgang mit dem Material sind sicherlich die Künstler. Wenn berichtet wird, dass Michelangelo den David aus einem Marmorblock schlägt, an dem sich zuvor mehrere andere vergeblich versucht hatten, und dann auf die Frage, wieso ihm gelingt, was andere nicht fertig gebracht haben, antwortet, er habe den David bloß aus seiner Verborgenheit im Marmorblock herausgeholt, den Marmor also gar nicht wirklich eigens gestaltet, dann ist damit genau dies gemeint: Michelangelo lebt so sehr im Marmor, dass er die im Marmor liegenden Seinsmöglichkeiten zu entdecken vermag.

„Die Sachen selber kommen jedoch nur darum weiter, weil einige [Menschen, N.W.] sind, die wirklich in den Dingen zu Hause sind und sie über die entscheidenden Strecken und Punkte weitertreiben. Sie treiben die Dinge weiter, weil sie diese aus ihren inneren Möglichkeiten heraus zu steigern verstehen und in der Weise der ,Konkreativität' neue Seinsmöglichkeiten hervortreiben, Seinsmöglichkeiten, die zugleich Möglichkeiten menschlichen Daseins und Möglichkeiten dinglicher Zusammenhänge sind. Die Einheit dieser Möglichkeiten ergibt sich durchaus nicht, wie Heidegger meint, , durch den Rückschlag der dinglichen Möglichkeiten ins Existenzverständnis des Daseins, sondern durch den Vor-ruck, den ein konkreativer Zugriff, der in eins Handlung und Erfahrung ist, bringt. “32

Welt geht daran auf, dass der Mensch sich so auf das Seiende einzulassen vermag, dass er neue Seinsmöglichkeiten dieses Seienden entdeckt, von denen 
her sich auch alles andere neu darstellt und die zugleich auch den Menschen selbst über sich hinausreißen und in die aufgehende Welt hineinziehen. Damit bin ich wieder am Anfang meines Textes. Dort habe ich gesagt: Das Zwischen meint jenen Ort, der zwischen dem Sichtbaren und dem, was im Sichtbaren sichtbar wird, zwischen Sein und Seiendem liegt. Dieses Zwischen ist der Ort des Wirklichkeitsgeschehens, in ihm gehören das Sichtbare und sein Sinn, Sein und Seiendes unmittelbar zusammen, und deswegen lässt sich das Sichtbare nur von ihm her in seiner eigentlichen Wirklichkeit sehen. Dort, wo es gelingt, in dieses Zwischen zu gelangen, geht Welt auf, so lässt sich nun sagen. Oder, um nochmals auf Heideggers eingangs zitierte Erläuterung von Phänomen zurückzukommen, etwas kann sich nur dann von sich her als das zeigen, was es ist, wenn an ihm Welt aufgeht und es in seiner Welt gesehen wird.

Welt gibt es deshalb nur im Plural. Die eine Welt ist bloße Abstraktion. Das hat Husserl bereits gesehen, wenn er von der einen Welt als einer bloßen Idee spricht und zeigt, dass die Naturwissenschaften ihre Vorstellung von der einen, objektiven Welt durch Idealisierung der geschichtlich realen Lebenswelt gewinnen. Das hat auch Heidegger gesehen, wenn er in Sein und Zeit von der „Jemeinigkeit“ von Welt spricht bzw. später auf die epochale Geschichtlichkeit von Welt aufmerksam macht. Rombach schließlich denkt die Pluralität von Welt ganz konsequent. Alles Sich-zeigen von etwas ist der Aufgang von Welt. Überall, wo sich etwas zeigt, geht Welt auf, d.h. es zeigt sich eigentlich nie etwas anderes als Welt. Welt ist das einzige Phänomen. Aber eben nicht so, dass dadurch alle Phänomene nivelliert würden, sondern umgekehrt gerade so, dass der Weltcharakter eines jeden Phänomens deutlich wird. Es gibt unzählig viele verschiedene Welten. Wir leben freilich üblicherweise nur in unserer eigenen und vermögen all die anderen Welten nicht zu entdecken. Bzw. noch schlimmer, wir leben noch nicht einmal in unserer eigenen Welt, sondern in der vermeintlich allgemeinen, von allen Erfahrungen absehenden und deshalb eben abstrakten Welt. Aufbrechen lässt sich diese abstrakte Welt nur dann, wenn wir sensibel werden für die unentdeckten Seinsmöglichkeiten, die in allem stecken. Wenn wir also zu Künstlern werden, die ihr Weltverstehen in der Schwebe zu halten und die Welt ebenso wie sich selbst in ihrem Umgang mit den Dingen immer wieder neu zu gewinnen vermögen. Das gilt auch für das epochale Weltverständnis.

„Die Daseinshermeneutik wird [in Heideggers Spätphilosophie, N.W.] nur in eine Seinshermeneutik gewendet, aber nicht in eine Wirklichkeitshermetik gehoben. Die Wirklichkeitshermetik würde erst dann in die denkerische Reichweite gelangen, wenn die jeweilige ,Lichtung' nicht vom Sein her, geschickt' würde, sondern aus der geschichtlichen Findung hervorkäme, mit der ein Volk eine neue Lebensmöglichkeit, eine neue Lebensdimension, eben eine ,Welt' aus sich und der Wirklichkeit hervortreibt." ${ }^{\text {33 }}$

Heinrich Rombach, Der kommende Gott. Hermetik - eine neue Weltsicht, Rombach, Freiburg 1991, S. 95. 


\title{
Niels Weidtmann
}

\section{Svijet kao fenomen}

\section{Komentar o Husserlu, Heideggeru i Rombachu}

\section{Sažetak}

Pisati istraživanje o svijetu kao fenomenu implicira pretpostavku da je svijet fenomen u smislu filozofske fenomenologije. To, međutim, nije jednoznačno čak ni unutar same fenomenologije. $U$ duhu husserlovska razumijevanja, svijet ne može biti ili postati fenomen zato što se svaki fenomen referira na relacijski sadržaj, tj. horizont. I dok bilo koji horizont može postati fenomen, zato što se referira na uvijek drugi horizont, svijet to ne može. Svijet je krajnji horizont i time se nema na što referirati. Dakle, nije samorazumljivo da se svijet uopće može uzeti kao fenomen. Međutim, u ovom radu polazim onkraj ove tvrdnje i argumentiram da je svijet fenomen par excellence. Takoreći, svijet je jedini fenomen uopće, ne samo zato što svemu što se u njemu pojavljuje daje poseban smisao nego i zato što se istovremeno sam pokazuje drugačijim, temeljem svojeg posebnog smisla. Pokušat ću navedeno demonstrirati referirajući se posebno na fenomenološke pristupe Heideggera i Rombacha. Čineći to, nadam se da ću ponuditi makar nekoliko aspekata fenomenološke analize »svijeta«.

\section{Ključne riječi}

svijet, smisao, fenomenologija, Edmund Husserl, Martin Heidegger, Heinrich Rombach, Maurice Merleau-Ponty

\section{Niels Weidtmann}

\section{World as Phenomenon}

\section{Commentary on Husserl, Heidegger and Rombach}

\begin{abstract}
To write a paper about the world as being a phenomenon implicates the assumption that the world is a phenomenon in the sense of philosophical phenomenology. That, however, is not uncontested even within phenomenology itself. In a Husserlian understanding, the world cannot be or become a phenomenon because any phenomenon refers to a relational context, i.e. to a horizon. While any horizon may become a phenomenon, because it refers to yet another horizon, the world cannot. World is the outmost horizon and, thus, it cannot refer to anything else. Therefore, it is not at all self-evident to claim that the world may be taken as a phenomenon. However, in my paper, I will go even beyond this claim and argue that world is, in fact, the phenomenon par excellence. In a sense, the world is the only phenomenon at all, because it not only gives its special meaning to everything that appears in the world, it itself appears differently, according to this special meaning. I will try to demonstrate this by referring particularly to the phenomenological approaches of Heidegger and Rombach. In the course of doing so, I hope to provide at least a few aspects of a phenomenological analysis of "world".
\end{abstract}

Keywords

world, meaning, phenomenology, Edmund Husserl, Martin Heidegger, Heinrich Rombach, Maurice Merleau-Ponty

\section{Niels Weidtmann}

\section{Le monde comme phénomène}

\section{Commentaires sur Husserl, Heidegger et Rombach}

\section{Résumé}

Écrire un article sur le monde en tant que phénomène implique l'hypothèse que le monde est un phénomène au sens de la phénoménologie philosophique. Mais cela n'est pas incontesté dans la phénoménologie elle-même. Selon la conception husserlienne, le monde ne peut pas être un 
phénomène en soi, car chaque phénomène apparaît dans un contexte de référence, c'est-à-dire dans un horizon, mais pas le monde lui-même. Le monde est l'horizon le plus éloigné et ne peut donc pas se référer au-delà de lui-même à un horizon plus large. Il n'est donc pas du tout évident d'affirmer que le monde peut être considéré comme un phénomène. Dans ma contribution, cependant, $j$ 'irai au-delà de l'hypothèse selon laquelle le monde est un phénomène et j'essaierai de montrer qu'en réalité, c'est le phénomène par excellence. D'une certaine manière, le monde est le seul phénomène de tous, car non seulement il donne son sens particulier à tout ce qui y apparaît, mais en même temps il se manifeste différemment, selon ce sens particulier. C'est ce que je veux essayer de montrer avec Heidegger et Rombach en particulier. J'espère ainsi révéler au moins quelques aspects d'une analyse phénoménologique du «monde».

\section{Mots-clés}

monde, Sens, phénoménologie, Edmund Husserl, Martin Heidegger, Heinrich Rombach, Maurice Merleau-Ponty 